\title{
Regularity of Extremal Solutions to Nonlinear Elliptic Equations with Quadratic Convection and General Reaction
}

Asadollah Aghajani, Fatemeh Mottaghi and Vicenţiu D. Rădulescu(i)

\begin{abstract}
We consider the nonlinear elliptic equation with quadratic convection $-\Delta u+g(u)|\nabla u|^{2}=\lambda f(u)$ in a smooth bounded domain $\Omega \subset \mathbb{R}^{N}(N \geq 3)$ with zero Dirichlet boundary condition. Here, $\lambda$ is a positive parameter, $f:[0, \infty):(0 \infty)$ is a strictly increasing function of class $C^{1}$, and $g$ is a continuous positive decreasing function in $(0, \infty)$ and integrable in a neighborhood of zero. Under natural hypotheses on the nonlinearities $f$ and $g$, we provide some new regularity results for the extremal solution $u^{*}$. A feature of this paper is that our main contributions require neither the convexity (even at infinity) of the function $h(t)=f(t) e^{-\int_{0}^{t} g(s) d s}$, nor that the functions $g h / h^{\prime}$ or $h^{\prime \prime} h / h^{\prime 2}$ admit a limit at infinity.
\end{abstract}

Mathematics Subject Classification. 35J91 (Primary), 35B09, 35B35, 35B65, 58J55 (Secondary).

Keywords. Nonlinear elliptic equation, extremal solution, stable condition, regularity, quadratic convection.

\section{Introduction and Main Result}

This article is devoted to the regularity properties of extremal solutions of the nonlinear Dirichlet elliptic equation with quadratic convection:

$$
\left\{\begin{array}{cc}
-\Delta u+g(u)|\nabla u|^{2}=\lambda f(u), & \text { in } \Omega, \\
u>0, & \text { in } \Omega, \\
u=0, & \text { on } \partial \Omega,
\end{array}\right.
$$

where $\Omega \subset \mathbb{R}^{N}(N \geq 3)$ is a smooth bounded domain, $\lambda$ is a positive real parameter, and $f$ is a $C^{1}$ strictly increasing function in $[0, \infty), f(0)>0$, and 
$g$ is a positive function, continuous either in $(0, \infty)$ or in $[0, \infty)$, decreasing and integrable in a neighborhood of zero.

The typical examples for the nonlinearity $f$ with the above properties are $(1+u)^{p}$ with $p>1$ and the exponential $e^{u}$. We can also include functions with linear growth at infinity, see Mironescu and Rădulescu [33,34]. Also for positive decreasing function $g$ in $(1)$, one can take $g(s)=s^{-\gamma}$ with $\gamma \in(0,1)$ as an example.

A positive function $u \in W_{0}^{1,2}(\Omega)$ is a weak solution of (1) if both $g(u)|\nabla u|^{2}$ and $f(u)$ belong to $L^{1}(\Omega)$ and for all $\phi \in W_{0}^{1,2}(\Omega) \cap L^{\infty}(\Omega)$ :

$$
\int_{\Omega} \nabla u \cdot \nabla \phi d x+\int_{\Omega} g(u)|\nabla u|^{2} \phi d x=\int_{\Omega} \lambda f(u) \phi d x .
$$

A solution $u$ of problem $(1)$ is said to be stable if $\left(f^{\prime}(u)-g(u) f(u)\right) \in L_{l o c}^{1}(\Omega)$ and for every $\phi \in W_{0}^{1,2}(\Omega)$ :

$$
\int_{\Omega}|\nabla \phi|^{2} d x \geq \lambda \int_{\Omega}\left(f^{\prime}(u)-g(u) f(u)\right) \phi^{2} d x .
$$

This condition was introduced by Arcoya [8]. Moreover, a solution $u$ of (1) is said to be regular if $u \in L^{\infty}(\Omega)$, and minimal if $u \leq v$ a.e in $\Omega$ for any other solution $v$, see Molino [35].

Quasilinear problems having lower order terms with quadratic growth with respect to the gradient play a crucial role in the study of nonlinear differential equations as they arise naturally in calculus of variations, stochastic control $[11,31]$ and motivated by wide applications such as thermal self-ignition in combustion theory and temperature distribution in an object heated by uniform electronic current, see [29,30,32].

Quasilinear Dirichlet problems of the type:

$$
\left\{\begin{array}{rr}
-\Delta u+g(u)|\nabla u|^{2}=f(x, u), & \text { in } \Omega, \\
u>0, & \text { in } \Omega, \\
u=0, & \text { on } \partial \Omega,
\end{array}\right.
$$

in the case when the right-hand side is not depending on $u$ have been extensively studied in the pioneering works by Boccardo et al. $[9,10,12]$, but the case when $f$ is nonlinear has been less studied. Arcoya et al. $[3-5,7]$ considered Problem (3) in the case when $g$ has singularity and described some applications. Moreover, some results about uniqueness, comparison, and maximum principles for the general form of the quasilinear elliptic equations with quadratic growth conditions have been proved in [15]. Orsina and Puel [37] considered Problem (3) where $g$ is a non-negative continuous function and proved several existence results for positive solutions of (3) with a power-like right-hand side. This case has been also studied recently by Boccardo et al. $[13,14]$. Furthermore, it is shown that if $g(u)=(1-u)^{-\gamma}$ in $(3)$ where $\gamma>0$, then the existence and non-existence of the solutions depend on the nonlinearity $f$ and the value of $\gamma$. It is worth mentioning here that some related problems are considered in [23-28] where the authors established several results related to existence, non-existence, or bifurcation of positive solutions for the boundary value problem $-\Delta u+K(x) g(u)+|\nabla u|^{a}=\lambda f(x, u)$ in $\Omega$, 
$u=0$ on $\partial \Omega$, where $\Omega$ is a smooth bounded domain, $0<a \leq 2, \lambda$ is a positive parameter, and $f$ is smooth and has a sublinear growth.

Arcoya et al. [8] proved that in Problem (1), if $f^{\prime}(s)-g(s) f(s)$ is an increasing function, such that $1 / f \in L^{1}(0, \infty)$ and there is a positive constant $c$ so that $\left|f^{\prime}(s) / f^{2}(s)\right| \leq c(1+\sqrt{g(s)})$, then there exists a parameter $\lambda^{*} \in$ $(0,+\infty)$, such that Problem (1) has a bounded minimal solution for $\lambda<\lambda^{*}$ and no solution for $\lambda>\lambda^{*}$. Furthermore, they proved that, under suitable conditions, the sequence of bounded minimal solutions for $\lambda<\lambda^{*}$ converges to a weak solution of Problem (1) for $\lambda=\lambda^{*}$, which is also stable and minimal. Molino [35] proved that if:

(H1) $\lim \sup _{s \longrightarrow \infty} g(s)<\infty$,

(H2) $f^{\prime}(s)-g(s) f(s)>0$ and non-singular $(s \geq 0)$,

(H3) $e^{-G(s)} \in L^{1}(1, \infty)$, where $G(s):=\int_{0}^{s} g(t) d t$,

(H4) $\forall C>0, \exists \tilde{C}>0: g(C s) \leq \tilde{C} g(s), \forall s<1$,

then there exists $\lambda^{*} \in(0,+\infty]$, such that for every $\lambda<\lambda^{*}$, there is a bounded minimal solution $u_{\lambda}$ of (1) and no solution for $\lambda>\lambda^{*}$. Also, the family of functions $\left\{u_{\lambda}\right\}_{0<\lambda<\lambda^{*}}$ is increasing and bounded in $W_{0}^{1,2}(\Omega)$ when the functions $f$ and $g$ satisfy the following extra condition:

$$
\lim _{s \longrightarrow \infty} \frac{s\left(f^{\prime}(s)-g(s) f(s)\right)}{f(s)}=\tau \in(1, \infty] .
$$

Moreover, it is proved that the increasing pointwise limit $u^{*}(x)=$ $\lim _{\lambda \longrightarrow \lambda^{*}} u_{\lambda}(x)$ is a weak solution of (1) for $\lambda=\lambda^{*}$, which is called the extremal solution. Furthermore, under conditions (H1)-(H5), if $f^{\prime}(s)-g(s) f(s)$ is a strictly increasing function, then every stable solution of Problem (1) is minimal. In particular, the extremal solution $u^{*}$ is stable and minimal, Molino [35].

We raise the following natural question: when is the extremal solution regular? Arcoya, Carmona, and Martínez-Aparicio [8] proved that if:

$$
\alpha:=\lim _{s \rightarrow \infty} \frac{g(s) f(s)}{f^{\prime}(s)} \quad \text { and } \quad \mu:=\lim _{s \rightarrow \infty} \frac{f(s)\left[f^{\prime}(s)-g(s) f(s)\right]^{\prime}}{f^{\prime}(s)\left[f^{\prime}(s)-g(s) f(s)\right]},
$$

then the extremal solution of Problem (1) is bounded whenever:

$$
N<4(1-\alpha)+2 \mu+4 \sqrt{\mu(1-\alpha)} .
$$

Also (see [8, Remark 4.8]), if $g \geq 0$ and for some $p, k>1, f(s) \sim k s^{p}$ for $s \gg 1$, then the above result can be improved to:

$$
N<\frac{p}{p-1}(4(1-\alpha)+2 \mu+4 \sqrt{\mu(1-\alpha)}) .
$$

As a particular case, if $f(u)=(1+u)^{p}$ and $g(u)=\frac{m}{1+u}$ in (1), where $m$ is a positive constant and $p>m+\frac{1}{m+1}$, then $u^{*}$ is regular whenever:

$$
3 \leq N<4\left(\frac{p-m}{p-1}\right)+2+4 \sqrt{\frac{p-m}{p-1}} .
$$

Molino [35] considered Problem (1) with $f(s)=e^{G(s)} h(s)$, where $h(s)$ is a differentiable function in $[0, \infty)$ and $h(0)>0$, and improved the results un- 
der assumptions (H1)-(H5). He proved that the extremal solution of Problem (1) (if $h$ is convex) is regular whenever:

$$
N<\frac{4+2(\tilde{\mu}+\tilde{\alpha})+4 \sqrt{\tilde{\mu}+\tilde{\alpha}}}{1+\tilde{\alpha}},
$$

where

$$
\tilde{\alpha}:=\lim _{s \longrightarrow \infty} \frac{g(s) h(s)}{h^{\prime}(s)} \text { and } \tilde{\mu}:=\lim _{s \longrightarrow \infty} \frac{h^{\prime \prime}(s) h(s)}{h^{\prime}(s)^{2}} .
$$

Remark 1. Notice that, in (4), we always have $\tilde{\mu} \geq 1-\frac{1}{\tau}>0$, where $\tau \in(1, \infty]$ defined in $(H 5)$, which also implies that the function $h$ must be eventually strictly convex and $f^{\prime}(s)-f(s) g(s)$ an eventually increasing function. To see this, take an arbitrary $\mu>\tilde{\mu}$, then, by the definition of $\tilde{\mu}$, there exists $s_{\mu}>0$, so that $\frac{h^{\prime \prime}(s)}{h^{\prime}(s)}<\mu \frac{h^{\prime}(s)}{h(s)}$ for all $s>s_{\mu}$. Then, by integrating twice we get $h(s)<C_{1} t^{\frac{1}{1-\mu}}$ for $t$ large, where $C_{1}$ is a positive constant. On the other hand, from $(H 5)$, for an arbitrary $\tau \in(1, \alpha)$, there exists $s_{\tau}>0$, such that $\frac{h^{\prime}(s)}{h(s)}>\frac{\tau}{s}$ for all $s>s_{\tau}$, implies that $h(s)>C_{2} s^{\tau}$ for all $s>s_{\tau}$, where $C_{2}$ is a positive castanet. Thus, we must have $\mu \geq 1-\frac{1}{\tau}$ that proves the claim. Moreover, notice that, by hypothesis (H2) on $f$ and $g$, the function $h$ is increasing, and as we have seen in the above, $h$ is also a superlinear function (that is, $\lim _{s \rightarrow \infty} \frac{h(s)}{s} \rightarrow \infty$ ).

It is worth mentioning here that there is a large literature devoted to the semi-linear analogue of (1), namely the Gelfand problem:

$$
\left\{\begin{aligned}
-\Delta u=\lambda f(u), & \text { in } \Omega, \\
u \geq 0, & \text { in } \Omega, \\
u=0, & \text { on } \partial \Omega,
\end{aligned}\right.
$$

where $\Omega \subset \mathbb{R}^{N \geq 1}$ is a smooth bounded domain, $\lambda$ is a positive parameter, and $f:[0, \infty] \longrightarrow \mathbb{R}$ is $C^{1}$, non-decreasing, superlinear and $f(0)>0$. Regularity of the extremal solutions of (5) has been extensively studied in the literature, and it is shown that it depends extremely on the dimension $N$, domain $\Omega$ and the nonlinearity $f$; see, for example $[1,2,17,19-22,36]$. It is proved that when $f(s)=e^{s}$, the extremal solution $u^{*}$ of $(5)$ is regular for $N<10$; also if $f(s)=$ $(1+s)^{p}$ and $p>1$, then $u^{*}$ is regular for $N<4+2(1-1 / p)+4 \sqrt{1-1 / p}$. Then, it was conjectured (related to two open problems stated by Brezis [16] in the context of "extremal solutions") that $u^{*}$ is bounded in dimension $N \leq 9$, and also belongs to the natural energy space $W_{0}^{1,2}(\Omega)$ in every dimension. Very recently, Cabré et al. [18] completely solved these two open problems and proved that stable solutions to semi-linear elliptic equations are bounded (and thus smooth) in dimension $N \leq 9$.

In this work, we consider Problem (1) with $f$ belongs to a general class of functions. At first, for the remainder of this paper, we set:

$$
h(s):=f(s) e^{-G(s)} .
$$


Then, we see that Problem (1) can be rewritten as:

$$
\left\{\begin{array}{cc}
-\Delta u+g(u)|\nabla u|^{2}=\lambda e^{G(u)} h(u), & \text { in } \Omega, \\
u>0, & \text { in } \Omega, \\
u=0, & \text { on } \partial \Omega,
\end{array}\right.
$$

and a solution $u$ of $(7)$ is stable if $e^{G(u)} h^{\prime}(u) \in L_{l o c}^{1}(\Omega)$ and satisfies:

$$
\int_{\Omega}|\nabla \phi|^{2} \geq \lambda \int_{\Omega} e^{G(u)} h^{\prime}(u) \phi^{2},
$$

for all $\phi \in W_{0}^{1,2}(\Omega)$.

In this case, hypotheses $\left(H_{2}\right)$ and $\left(H_{5}\right)$ take the following simple forms, respectively:

$$
h^{\prime}(t)>0 \text {, and is non-singular for } s \geq 0 \text { and } \lim _{s \rightarrow \infty} \frac{s h^{\prime}(s)}{h(s)}=\tau \in(1, \infty] .
$$

As we mentioned in Remark (1), all previous works assume that $h$ is a convex function (or eventually convex). However, in this paper, we remove this extra restriction and allow the function $h$ to be nonconvex. Thus, instead of the parameters like as (4) used in previous works, we define the following new ones:

$$
\begin{aligned}
& \alpha_{-}:=\liminf _{t \longrightarrow \infty} \frac{h^{\prime}(t) H(t)}{h(t)^{2}} \leq \alpha_{+}:=\limsup _{t \longrightarrow \infty} \frac{h^{\prime}(t) H(t)}{h(t)^{2}}, \\
& \beta_{-}:=\liminf _{t \longrightarrow \infty} \frac{g(t) H(t)}{h(t)} \leq \beta_{+}:=\limsup _{t \longrightarrow \infty} \frac{g(t) H(t)}{h(t)}
\end{aligned}
$$

where $H(t):=\int_{0}^{t} h(s) d s$. Now, we state our main regularity result.

Theorem 1. Let $u^{*}$ be the extremal solution of Problem (7) and $\Omega$ be an arbitrary bounded smooth domain. If $0<\alpha_{-} \leq \alpha_{+}<\infty, \beta_{+}<\infty$ and $2 \alpha_{-}+\beta_{-} \geq 1$, then $u^{*} \in L^{\infty}(\Omega)$ whenever:

$$
N<\frac{4 \alpha_{-}}{\alpha_{-}+\beta_{+}}\left(1+\frac{\sqrt{\alpha_{-}\left(2 \alpha_{-}+\beta_{-}-1\right)}}{\alpha_{+}}+\frac{2 \alpha_{-}+\beta_{-}-1}{2 \alpha_{+}}\right) .
$$

If, in addition to the above assumptions, we have $\alpha_{-}+\beta_{-} \leq 1$, then $u^{*} \in$ $L^{\infty}(\Omega)$ whenever:

$$
N<\frac{4 \alpha_{-}}{\alpha_{-}+\beta_{+}}\left(1+\sqrt{\frac{2 \alpha_{-}+\beta_{-}-1}{\alpha_{+}}}+\frac{2 \alpha_{-}+\beta_{-}-1}{2 \alpha_{+}}\right) .
$$

\subsection{Examples}

We provide several examples of functions that fulfill the above hypotheses.

Example 1. Consider Problem (1) with $g(t) \equiv C$ and $f(t)=e^{\gamma t}(\gamma>C>0$ ), which is equivalent to Problem (7) with $h(t)=e^{(\gamma-C) t}$. Here, it is easy to see that we have $\alpha_{+}=\alpha_{-}=1$ and $\beta_{+}=\beta_{-}=\frac{C}{\gamma-C}$. Then, by (9) in Theorem $1, u^{*} \in L^{\infty}(\Omega)$ whenever:

$$
N<6-4 \frac{C}{\gamma}+4 \sqrt{1-\frac{C}{\gamma}}
$$


We remark that for a fixed $\gamma>0$, by letting $C \longrightarrow 0$, the right-hand side of (11) goes to 10 which gives the optimal regularity dimension $N \leq 9$ for the extremal solution of the limit equation [Eq. (5)], with the exponential nonlinearity. Furthermore, the above result coincides with Proposition 3.2 in [39].

Also with the above function $g \equiv C$ where $C$ is a positive constant, if we set $h(t):=e^{t}(1+0.1 \sin t)$ in Problem $(7)$, then we observe that $h$ satisfies the needed assumptions and also:

$$
\begin{gathered}
\frac{h^{\prime}(t) H(t)}{h(t)^{2}}=\frac{(1+0.1 \cos t+0.1 \sin t)(1+0.05 \sin t-0.05 \cos t)}{(1+0.1 \sin t)^{2}}, \\
\frac{g(t) H(t)}{h^{\prime}(t)}=\frac{C(1+0.05 \sin t-0.05 \cos t)}{1+0.1 \sin t},
\end{gathered}
$$

which are periodic functions with period $2 \pi$. By Mathematica, we compute:

$$
\begin{aligned}
& \alpha_{-}=\min _{[0,2 \pi]} \alpha(u) \approx 0.933238, \quad \alpha_{+}=\max _{[0,2 \pi]} \alpha(u) \approx 1.07681, \\
& \beta_{-}=\min _{[0,2 \pi]} \beta(u) \approx 0.9338 C \quad \text { and } \quad \beta_{+}=\max _{[0,2 \pi]} \beta(u) \approx 1.0761 C .
\end{aligned}
$$

Then, by (9) in Theorem 1 , we see that the extremal solution $u^{*} \in L^{\infty}(\Omega)$ whenever:

$$
N<\frac{3.7329}{0.9332+1.0761 C}\left(1+\frac{\sqrt{0.9332(0.8664+0.9338 C)}}{1.0768}+\frac{0.8664+0.9338 C}{2.1536}\right) .
$$

However, if $C<\frac{1}{9}$, then $\alpha_{-}+\beta_{-}<1$ and by (10) in Theorem 1 , we can get a better upper bound for the regularity dimension for $u^{*}$, that is:

$$
N<\frac{3.7329}{0.9332+1.0761 C}\left(1+\sqrt{\frac{0.8664+0.9338 C}{1.0768}}+\frac{0.8664+0.9338 C}{2.1536}\right) .
$$

Again note that by the above result, if $C$ is sufficiently small, then $u^{*}$ is regular for $N \leq 9$.

Example 2. Consider Problem (7) with $g(t)=\frac{\delta}{t+1}$, where $\delta$ is a positive constant and $h(t)=\left(t^{2}+3 t+3 \cos t+4\right)$. It is not hard to see that $h$ satisfies all the needed assumptions, but is not convex even at infinity (and, hence, none of the previous results apply). However, we easily see that $\beta_{+}=\beta_{-}=\frac{\delta}{3}$ and $\alpha_{+}=\alpha_{-}=\frac{2}{3}$, and then, by Theorem 1, the extremal solution $u^{*}$ of Problem (7) is bounded when:

$$
N<\frac{8}{2+\delta}\left(1+\sqrt{\frac{\delta+1}{2}}+\frac{\delta+1}{4}\right) .
$$

Example 3. Consider Problem (7) with $g(t)=\frac{1}{t^{\gamma}}$, where $\gamma \geq 1$ and $h(t)=$ $e^{t}(15+8 \sin t)$. Here, $h$ is increasing but not convex. Indeed, we have $h^{\prime \prime}(t)=$ $e^{t}(15-16 \cos t)$, and hence, $\liminf _{t \rightarrow \infty} h^{\prime \prime}(t)=-\infty$. However:

$$
\frac{h^{\prime}(t) H(t)}{h(t)^{2}}=\frac{(15+4 \sin t-4 \cos t)(15+8 \sin t+8 \cos t)}{(15+8 \sin t)^{2}},
$$


which is a periodic function with period $2 \pi$. By Mathematica, we can compute that:

$$
\alpha_{-}=\min _{[0,2 \pi]} \alpha(t) \approx 0.547593 \quad \text { and } \quad \alpha_{+}=\max _{[0,2 \pi]} \alpha(t) \approx 1.80247 .
$$

Also, it is not hard to see that $\beta_{+}=\beta_{-}=0$. Then, by (10) in Theorem 1 , $u^{*}$ is bounded when $N<6$.

\section{Proof of the Main Result}

The next simple technical lemma and its companion Proposition 1 are key ingredients in the proof of our main result.

Lemma 1. Let $u_{\lambda}$ be the stable solution of (7). Also, $m:[0, \infty) \longrightarrow[0, \infty)$ is a $C^{1}$ function which is zero in a neighborhood of zero and satisfies:

$$
K(t):=e^{G(t)} h^{\prime}(t) m^{2}(t)-h(t) \int_{0}^{t} e^{G(s)} m^{\prime}(s)^{2} d s \geq 0, \quad \text { for } t \text { sufficiently large, }
$$

where $G(t)=\int_{0}^{t} g(s) d s$. Then, $\left\|K\left(u_{\lambda}\right)\right\|_{L^{1}(\Omega)} \leq C$, where $C$ is a constant independent of $\lambda$.

Proof. Let $u:=u_{\lambda}>0$ be the stable minimal solution of Problem (7). Taking $\phi=m(u)$ in the stability inequality (8), we obtain:

$$
\begin{gathered}
\int_{\Omega} \nabla M \cdot \nabla u d x \geq \lambda \int_{\Omega} e^{G(u)} h^{\prime}(u) m(u)^{2} d x \Longrightarrow \int_{\Omega}(-\Delta u) M(u) d x \geq \lambda \\
\int_{\Omega} e^{G(u)} h^{\prime}(u) m(u)^{2} d x,
\end{gathered}
$$

where $M(s):=\int_{0}^{s} m^{\prime}(t)^{2} d t$. Then, according to Eq (7), we obtain:

$$
\int_{\Omega}\left(\lambda e^{G(u)} h(u)-g(u)|\nabla u|^{2}\right) M(u) d x \geq \lambda \int_{\Omega} e^{G(u)} h^{\prime}(u) m^{2}(u) d x .
$$

On the other hand, $u$ is a weak solution of (7), and hence:

$$
\int_{\Omega} \nabla u \cdot \nabla \psi(x) d x+\int_{\Omega} g(u)|\nabla u|^{2} \psi(x) d x=\lambda \int_{\Omega} e^{G(u)} h(u) \psi(x) d x,
$$

for every $\psi \in W_{0}^{1,2}(\Omega)$. Set $\psi(x):=M(u(x))-e^{-G(u(x))} \int_{0}^{u(x)} e^{G(t)} m^{\prime}(t)^{2} d t$, $x \in \Omega$. Then, notice that we have $\psi(x)=0$ when $u(x)$ is near zero (by the assumption on the function $m$ ) and:

$$
\nabla \psi=g(u) e^{-G(u)}\left(\int_{0}^{u} e^{G(t)} m^{\prime}(t)^{2} d t\right) \nabla u .
$$

Therefore, since $\nabla \psi(x)=0$ when $u(x)$ is near zero and $g$ is continuous in $(0, \infty)$, we get $\psi \in W_{0}^{1,2}(\Omega)$. Now, we substitute $\psi$ in the above equality as a test function to get: 


$$
\begin{aligned}
\int_{\Omega} g(u) e^{-G(u)}\left(\int_{0}^{u} e^{G(t)} m^{\prime}(t)^{2} d t\right)|\nabla u|^{2} d x+\int_{\Omega} g(u) M(u)|\nabla u|^{2} d x \\
\quad-\int_{\Omega} g(u) e^{-G(u)}\left(\int_{0}^{u} e^{G(t)} m^{\prime}(t)^{2} d t\right)|\nabla u|^{2} d x \\
=\lambda \int_{\Omega} e^{G(u)} h(u) M(u) d x-\lambda \int_{\Omega} h(u)\left(\int_{0}^{u} e^{G(t)} m^{\prime}(t)^{2} d t\right) d x .
\end{aligned}
$$

Canceling the first and the third terms on the left of the equality above, we obtain:

$$
\int_{\Omega}\left(\lambda e^{G(u)} h(u)-g(u)|\nabla u|^{2}\right) M(u) d x=\int_{\Omega} h(u)\left(\int_{0}^{u} e^{G(t)} m^{\prime}(t)^{2} d t\right) d x .
$$

Using (14) in (13), we then arrive at:

$$
\left.\int_{\Omega} e^{G(u)} h^{\prime}(u) m^{2}(u) d x-\int_{\Omega} h(u)\left(\int_{0}^{u} e^{G(t)} m^{\prime}(t)^{2}\right) d t\right) d x \leq 0,
$$

Now, by the definition of the function $K$ given in (12), the inequality (15) can be read as:

$$
\int_{\Omega} K(u) d x \leq 0
$$

Now, by the assumption (12), there is an $s_{0}>0$ such that $K(s) \geq 0$, where $s \geq s_{0}$, and then, by (16), we can write:

$$
\begin{aligned}
\int_{\Omega}|K(u)| d x & =\int_{u \leq s_{0}}|K(u)| d x+\int_{u \geq s_{0}} K(u) d x \\
& \leq \int_{u \leq s_{0}}(|K(u)|-K(u)) d x \leq C_{0}|\Omega|,
\end{aligned}
$$

where $|\Omega|$ is the Lebesgue measure of $\Omega$ and $C_{0}:=\sup _{s \in\left[0, s_{0}\right]}(|K(u)|-K(u))$ which is independent of $u$ that proves the desired result.

Proposition 1. Let $u_{\lambda}$ be the stable solution of $(7)$ and $w:[0, \infty) \longrightarrow[0, \infty)$ be a $C^{1}$ function, such that, for some $t_{0}>0$, we have $w(t) \leq \frac{h^{\prime}(t)}{h(t)}, w^{2}(t)+$ $w^{\prime}(t)+g(t) w(t) \geq 0$ for $t \geq t_{0}$, where:

$$
E(t):=h(t)\left(\frac{h^{\prime}(t)}{h(t)}-w(t)\right) e^{G(t)} e^{2 \int_{t_{0}}^{t} w(s)+\sqrt{w^{2}(s)+w^{\prime}(s)+g(s) w(s)} d s},
$$

and $\frac{E(t)}{h(t)} \longrightarrow \infty$ as $t \longrightarrow \infty$. Then, $\left\|E\left(u_{\lambda}\right)\right\|_{L^{1}(\Omega)} \leq C$, where $C$ is a constant independent of $\lambda$.

Proof. Let $m:[0, \infty) \longrightarrow[0, \infty)$ be a $C^{1}$ function which is zero in a neighborhood of zero and:

$$
m(t)=e^{\int_{t_{0}}^{t} w(s)+\sqrt{w^{2}(s)+w^{\prime}(s)+g(s) w(s)} d s}, \text { for } t \geq t_{0},
$$


where $t_{0}$ and $w$ given in the statement of the proposition. Then, using the equality

$$
m^{\prime}(t)=m(t)\left[w(t)+\sqrt{w^{2}(t)+w^{\prime}(t)+g(t) w(t)}\right] \text { for } t \geq t_{0},
$$

we obtain:

$$
\begin{aligned}
& \frac{d}{d t}\left(w(t) m^{2}(t) e^{G(t)}-\int_{t_{0}}^{t} e^{G(s)} m^{\prime}(s)^{2} d s\right)= \\
& m^{2}(t) e^{G(t)}\left(w^{\prime}(t)+2 \frac{m^{\prime}(t)}{m(t)} w(t)+w(t) g(t)-\left(\frac{m^{\prime}(t)}{m(t)}\right)^{2}\right)=0,
\end{aligned}
$$

for all $t \geq t_{0}$. It follows that:

$$
\int_{t_{0}}^{t} e^{G(s)} m^{\prime}(s)^{2} d s=w(t) m(t)^{2} e^{G(t)}+C_{0},
$$

where $C_{0}$ is a constant. Now, by (18), for $t \geq t_{0}$, we have:

$$
K(t):=e^{G(t)} m(t)^{2} h^{\prime}(t)-h(t) \int_{0}^{t} e^{G(s)} m^{\prime}(s)^{2} d s=E(t)-C_{0} h(t),
$$

which is positive for large $t$ sufficiently large (by the assumption), and hence, by Lemma 1 , we get $\left\|K\left(u_{\lambda}\right)\right\|_{L^{1}(\Omega)} \leq C_{1}$, where $C_{1}$ is a constant independent of $\lambda$. And since, by the assumption and (19), we have:

$$
K(t)=E(t)\left[1-C_{0} \frac{h(t)}{E(t)}\right] \geq \frac{E(t)}{2} \text { for } t \text { sufficiently large, }
$$

we get also that $\left\|E\left(u_{\lambda}\right)\right\|_{L^{1}(\Omega)} \leq C_{2}$, where $C_{2}$ is a constant independent of $\lambda$, which is the desired result.

\subsection{Proof of Theorem 1}

We now give the proof of our main result. The strategy for the proof is to apply Proposition 1, by choosing a suitable function $w$ in term of the nonlinearity $h$, so that the corresponding function $E$ defined by (17) is comparable to some power of $h$, to get some $L^{p}$ estimates for $e^{G\left(u_{\lambda}\right)} h\left(u_{\lambda}\right)$ (the right-hand side of Eq. (7)) independent of $\lambda$, and then applying the standard regularity result by Stampacchia's lemma [38].

Assume that $\alpha_{+}, \beta_{+}<\infty$ and $2 \alpha_{-}+\beta_{-} \geq 1$. Take arbitrary $\alpha_{1}, \alpha_{2}, \alpha_{3}$, $\beta_{1}, \beta_{2}$, such that $\alpha_{1}<\alpha_{2}<\alpha_{-} \leq \alpha_{+}<\alpha_{3}$ and $\beta_{1}<\beta_{-} \leq \beta_{+}<\beta_{2}$. Then, by the definition of $\alpha_{ \pm}$and $\beta_{ \pm}$, we can find a $t_{0}>0$, so that for $t \geq t_{0}$ :

$$
\alpha_{1}<\alpha_{2}<\frac{h^{\prime}(t) H(t)}{h^{2}(t)}<\alpha_{3} \text { and } \beta_{1}<\frac{g(t) H(t)}{h(t)}<\beta_{2} \text {. }
$$

Let $w:[0, \infty) \longrightarrow[0, \infty)$ be a $C^{1}$ function, such that $w(t)=\alpha_{1} \frac{h(t)}{H(t)}$ for $t \geq t_{0}$, where $H(t)=\int_{0}^{t} h(s) d s$ as before. From (20), we have:

$$
g(t)>\frac{\beta_{1}}{\alpha_{1}} w(t) \text { and } \quad \frac{h^{\prime}(t)}{H(t)}>\alpha_{1} \frac{h(t)^{2}}{H(t)^{2}} .
$$


Thus, using these inequalities and the definition of $w$, we obtain:

$$
\begin{aligned}
& w^{2}(t)+w^{\prime}(t)+g(t) w(t) \geq \alpha_{1}\left[\frac{h^{\prime}(t)}{H(t)}+\left(\alpha_{1}+\beta_{1}-1\right) \frac{h(t)^{2}}{H(t)^{2}}\right] \\
& \geq \alpha_{1}\left(2 \alpha_{1}+\beta_{1}-1\right) \frac{h(t)^{2}}{H(t)^{2}}
\end{aligned}
$$

for $t \geq t_{0}$.

The inequalities in (20) imply that:

$$
g(t) \geq \frac{\beta_{1}}{\alpha_{3}} \frac{h^{\prime}(t)}{h(t)} \quad \text { and } \quad \frac{h^{\prime}(t)}{h(t)} \geq \alpha_{2} \frac{h(t)}{H(t)} \quad \text { for } t \geq t_{0} ;
$$

hence:

$$
e^{G(t)} \geq C h(t)^{\frac{\beta_{1}}{\alpha_{3}}} \quad \text { and } \quad \frac{h^{\prime}(t)}{h(t)}-w(t) \geq\left(\alpha_{2}-\alpha_{1}\right) \frac{h(t)}{H(t)}
$$

for $t \geq t_{0}$.

Let the function $E(t)$ be given as in (18) in Proposition 1. By the inequalities (20), (21), (22) and the fact that $\int_{t_{0}}^{t} w(s) d s=\alpha_{1}(\ln H(t)-$ $\left.\ln H\left(t_{0}\right)\right)$, we obtain:

$$
\begin{aligned}
E(t) & =h(t)\left(\frac{h^{\prime}(t)}{h(t)}-w(t)\right) e^{G(t)} e^{2 \int_{t_{0}}^{t} w(s)+\sqrt{w^{2}(s)+w^{\prime}(s)+g(s) w(s)} d s} \\
& \geq C h^{2+\frac{\beta_{1}}{\alpha_{3}}} H^{2 \alpha_{1}+2 \sqrt{\alpha_{1}\left(2 \alpha_{1}+\beta_{1}-1\right)}-1},
\end{aligned}
$$

where $C$ is a positive constant which depends only on $h$.

Now, writing the first inequality in $(20)$ as $\frac{h^{\prime}(t)}{h(t)}<\alpha_{3} \frac{h(t)}{H(t)}$ for $t_{0}>0$ and integrating from $t_{0}$ to $t$, we obtain:

$$
H(t)>C h(t)^{\frac{1}{\alpha_{3}}} \quad \text { for } t \geq t_{0} .
$$

Using the above inequality in (23), we arrive at:

$E(t) \geq h(t)^{\gamma}, \quad$ where $\quad \gamma:=2\left(1+\frac{\sqrt{\alpha_{1}\left(2 \alpha_{1}+\beta_{1}-1\right)}}{\alpha_{3}}+\frac{2 \alpha_{1}+\beta_{1}-1}{2 \alpha_{3}}\right)$,

for all $t \geq t_{0}$.

Since $\frac{E(t)}{h(t)} \longrightarrow \infty$ as $t \longrightarrow \infty$, then from Proposition 1 , we get $\left\|E\left(u_{\lambda}\right)\right\|_{L^{1}(\Omega)} \leq C$. Next, by $(25)$, we deduce that $\left\|h\left(u_{\lambda}\right)\right\|_{L^{\gamma}(\Omega)} \leq C$, where $C$ is a constant independent of $\lambda$. On the other hand, by (20), we have $g(t) \leq \frac{\beta_{2}}{\alpha_{1}} \frac{h^{\prime}(t)}{h(t)}$ for $t \geq t_{0}$. Thus, by integration over $\left[t_{0}, t\right]$, we obtain:

$$
e^{G(t)} h(t) \leq C h(t)^{\frac{\alpha_{1}+\beta_{2}}{\alpha_{1}}} \text { for } t \geq t_{0} .
$$

Therefore:

$$
\left\|e^{G\left(u_{\lambda}\right)} h\left(u_{\lambda}\right)\right\|_{L^{\mu}(\Omega)} \leq C
$$


for

$$
\mu:=\frac{\alpha_{1}}{\alpha_{1}+\beta_{2}} \gamma=\frac{2 \alpha_{1}}{\alpha_{1}+\beta_{2}}\left(1+\frac{\sqrt{\alpha_{1}\left(2 \alpha_{1}+\beta_{1}-1\right)}}{\alpha_{3}}+\frac{2 \alpha_{1}+\beta_{1}-1}{2 \alpha_{3}}\right),
$$

where $C$ is a constant independent of $\lambda$.

Note that $e^{G\left(u_{\lambda}\right)} h\left(u_{\lambda}\right)$ is the right-hand side of Eq. (7), and therefore, by Stampacchia's lemma [38], we obtain $u^{*} \in L^{\infty}(\Omega)$ for $N<2 \mu$. Since $\alpha_{1}, \alpha_{2}, \alpha_{3}, \beta_{1}, \beta_{2}$ were arbitrary (in the given ranges), we conclude that $u^{*} \in$ $L^{\infty}(\Omega)$ for:

$$
N<\frac{4 \alpha_{-}}{\alpha_{-}+\beta_{+}}\left(1+\frac{\sqrt{\alpha_{-}\left(2 \alpha_{-}+\beta_{-}-1\right)}}{\alpha_{+}}+\frac{2 \alpha_{-}+\beta_{-}-1}{2 \alpha_{+}}\right),
$$

which is the desired result in the first part of theorem.

To complete the proof, we assume that $\alpha_{-}+\beta_{-} \leq 1$. Thus, by the above notation and by the first inequality in (20), we obtain:

$$
\begin{aligned}
w^{2}(t)+w^{\prime}(t)+g(t) w(t) & \geq \alpha_{1}\left[\frac{h^{\prime}(t)}{H(t)}-\left(1-\alpha_{1}-\beta_{1}\right) \frac{h^{2}(t)}{H^{2}(t)}\right] \\
& \geq\left(2 \alpha_{1}+\beta_{1}-1\right) \frac{h^{\prime}(t)}{H(t)} \geq\left(\frac{2 \alpha_{1}+\beta_{1}-1}{\alpha_{3}}\right) \frac{h^{\prime}(t)^{2}}{h(t)^{2}}
\end{aligned}
$$

for all $t \geq t_{0}$.

Assuming that the function $E(t)$ is given as in (18) in Proposition 1, relation (27) together with estimates (20) and (22) yields:

$$
\begin{aligned}
E(t) & =h(t)\left(\frac{h^{\prime}(t)}{h(t)}-w(t)\right) e^{G(t)} e^{2 \int_{t_{0}}^{t} w(s)+\sqrt{w^{2}(s)+w^{\prime}(s)+g(s) w(s)} d s} \\
& \geq C h^{2+\frac{\beta_{1}}{\alpha_{3}}+2 \sqrt{\frac{2 \alpha_{1}+\beta_{1}-1}{\alpha_{3}}}} H^{2 \alpha_{1}-1} .
\end{aligned}
$$

Using now (24), we deduce that:

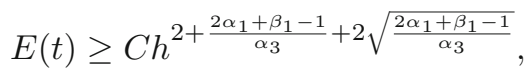

for $t \geq t_{0}$, where $C$ is a positive constant depends only on $h$.

Next, with similar arguments as in the first part and using the above inequality together with relations (24), (26), and Proposition 1, we get:

$$
\begin{aligned}
& \left\|e^{G\left(u_{\lambda}\right)} h\left(u_{\lambda}\right)\right\|_{L^{\theta}(\Omega)} \leq C \text { for } \theta \\
& \quad:=\frac{2 \alpha_{1}}{\alpha_{1}+\beta_{2}}\left(1+\sqrt{\frac{2 \alpha_{1}+\beta_{1}-1}{\alpha_{3}}}+\frac{2 \alpha_{1}+\beta_{1}-1}{2 \alpha_{3}}\right),
\end{aligned}
$$

where $C$ is a constant independent of $\lambda$, which implies that $u^{*} \in L^{\infty}(\Omega)$ for $N<2 \theta$. Again, since $\alpha_{1}, \alpha_{2}, \alpha_{3}, \beta_{1}, \beta_{2}$ were arbitrary (in the given ranges), we get $u^{*} \in L^{\infty}(\Omega)$ for:

$$
N<\frac{4 \alpha_{-}}{\alpha_{-}+\beta_{+}}\left(1+\sqrt{\frac{2 \alpha_{-}+\beta_{-}-1}{\alpha_{+}}}+\frac{2 \alpha_{-}+\beta_{-}-1}{2 \alpha_{+}}\right),
$$

which completes the proof of the theorem. 


\section{Acknowledgements}

The authors would like to thank the anonymous referee for a careful reading of the manuscript and helpful comments and suggestions.

Open Access. This article is licensed under a Creative Commons Attribution 4.0 International License, which permits use, sharing, adaptation, distribution and reproduction in any medium or format, as long as you give appropriate credit to the original author(s) and the source, provide a link to the Creative Commons licence, and indicate if changes were made. The images or other third party material in this article are included in the article's Creative Commons licence, unless indicated otherwise in a credit line to the material. If material is not included in the article's Creative Commons licence and your intended use is not permitted by statutory regulation or exceeds the permitted use, you will need to obtain permission directly from the copyright holder. To view a copy of this licence, visit http:// creativecommons.org/licenses/by/4.0/.

Publisher's Note Springer Nature remains neutral with regard to jurisdictional claims in published maps and institutional affiliations.

\section{References}

[1] Aghajani, A.: New a priori estimates for semistable solutions of semilinear elliptic equations. Potential Anal. 44, 729-744 (2019)

[2] Aghajani, A.: Regularity of extremal solutions of semilinear elliptic problems with non-convex nonlinearities on general domains. Discrete Contin. Dyn. Syst. Ser. A 37, 3521-3530 (2017)

[3] Arcoya, D., Barile, S., Martínez-Aparicio, P.J.: Singular quasilinear equations with quadratic growth in the gradient without sign condition. J. Math. Anal. Appl. 350, 401-408 (2009)

[4] Arcoya, D., Boccardo, L., Leonori, T., Porretta, A.: Some elliptic problems with singular natural growth lower order terms. J. Diff. Equ. 249(11), 27712795 (2010)

[5] Arcoya, D., Carmona, J., Leonori, T., Martinez-Aparicio, P.J., Orsina, L., Petitta, F.: Existence and nonexistence of solutions for singular quadratic quasilinear equations. J. Diff. Equ. 246(10), 4006-4042 (2009)

[6] Arcoya, D., Carmona, J., Martínez-Aparicio, P.J.: Bifurcation for quasilinear elliptic singular BVP. Comm. Partial Diff. Equ. 36(4), 670-692 (2011)

[7] Arcoya, D., Segura de León, S.: Uniqueness of solutions for some elliptic equations with a quadratic gradient term. ESAIM Control Opt. Calc. Var. 16(2), 327-336 (2010)

[8] Arcoya, D., Carmona, J., Martínez-Aparicio, P.J.: Gelfand type quasilinear elliptic problems with quadratic gradient terms. Ann. Inst. H. Poincaré - Analyse Non Linéaire 31, 249-265 (2014)

[9] Boccardo, L., Murat, F., Puel, J.P.: Existence of unbounded solutions for some quasilinear equations. Portugaliae Math. 41, 507-534 (1982)

[10] L. Boccardo, F. Murat, J.P. Puel, Existence de solutions faibles pour des équations elliptiques quasi-liéaires á croissance quadratique, in Nonlinear Partial Differential Equations and Their Applications Collège De France Seminar, 
Vol. IV (J.-L. Lions and H. Brézis, eds.), Research Notes in Math, 84, Pitman, London, pp. 19-73, 1983

[11] Boccardo, L.: Dirichlet problems with singular and gradient quadratic lower order terms. ESAIM Control Opt. Calc. Var. 14, 411-426 (2008)

[12] Boccardo, L., Murat, F., Puel, J.P.: Existence of bounded solutions for nonlinear elliptic unilateral problems. Ann. Mat. Pura Appl. 152(4), 183-196 (1988)

[13] Boccardo, L., Orsina, L., Porzio, M.M.: Existence results for quasilinear elliptic and parabolic problems with quadratic gradient terms and sources. Adv. Calc. Var. 4(4), 397-419 (2011)

[14] Boccardo, L., Leonori, T., Orsina, L., Petitta, F.: Quasilinear elliptic equations with singular quadratic growth terms. Comm. Contemp. Math. 13(4), 607-642 (2011)

[15] Barles, G., Murat, F.: Uniqueness and the maximum principle for quasilinear elliptic equations with quadratic growth conditions. Arch. Ration. Mech. Anal. 133, 77-101 (1995)

[16] Brezis, H., Is there failure of the inverse function theorem?, Morse theory, minimax theory and their applications to nonlinear differential equations, pp. 23-33, New Stud. Adv. Math., 1, Int. Press, Somerville, MA, (2003)

[17] Cabré, X., Capella, A.: Regularity of radial minimizers and extremal solutions of semilinear elliptic equations. J. Diff. Equ. 236(1), 164-198 (2007)

[18] Cabré, X., Figalli, A., Ros-Oton, X., Serra, J.: Stable solutions to semilinear elliptic equations are smooth up to dimension 9. Acta Math. 224(2), 187-252 (2020)

[19] Cabré, X., Sanchón, M.: Geometric-type Sobolev inequalities and applications to the regularity of minimizers. J. Funct. Anal. 264, 303-325 (2013)

[20] Cabré, X., Sanchón, M., Spruck, J.: A priori estimates for semistable solutions of semilinear elliptic equations. Discrete Contin. Dyn. Syst. Ser. A 36, 601-609 (2016)

[21] Crandall, M.G., Rabinowitz, P.H.: Some continuation and variational methods for positive solutions of nonlinear elliptic eigenvalue problems. Arch. Rational Mech. Anal. 58, 207-218 (1975)

[22] Dupaigne, L.: Stable Solutions to Elliptic Partial Differential Equations, Chapman and Hall-CRC Monographs and Surveys in Pure and Applied Mathematics, vol. 143. Chapman and Hall-CRC, Boca Raton (2011)

[23] Ghergu, M., Rădulescu, V.: Bifurcation for a class of singular elliptic problems with quadratic convection term, C. R. Acad. Sci. Paris, Sér. I 338, 831-836 (2004)

[24] Ghergu, M., Rădulescu, V.: On a class of sublinear singular elliptic problems with convection term. J. Math. Anal. Appl. 311, 635-646 (2005)

[25] Ghergu, M., Rădulescu, V.: Bifurcation and asymptotics for the Lane-EmdenFowler equation. C. R. Acad. Sci. Paris, Sér. I 337, 259-264 (2003)

[26] Ghergu, M., Rădulescu, V.: Nonradial blow-up solutions of sublinear elliptic equations with gradient term. Commun. Pure Appl. Anal. 3, 465-474 (2004)

[27] Ghergu, M., Rădulescu, V.: Multi-parameter bifurcation and asymptotics for the singular Lane-Emden-Fowler equation with a convection term. Proc. Roy. Soc. Edinburgh Sect. A 135(1), 61-83 (2005) 
[28] Ghergu, M., Rădulescu, V.: Singular elliptic problems: bifurcation and asymptotic analysis, Oxford Lecture Series in Mathematics and its Applications, 37. The Clarendon Press, Oxford University Press, Oxford (2008)

[29] Gel'fand, I.M.: Some problems in the theory of quasi-linear equations. (Russian) Uspehi Mat. Nauk 14(2), 87-158 (1959)

[30] Joseph, D., Lundgren, T.S.: Quasilinear Dirichlet problems driven by positive sources. Arch. Ration. Mech. Anal. 49, 241-269 (1973)

[31] Jerison, D., Monneau, R.: Towards a counter-example to a conjecture of De Giorgi in high dimensions. Ann. Mat. Pura Appl. 183(4), 439-467 (2004)

[32] Keller, H.B., Cohen, D.S.: Some positone problems suggested by nonlinear heat generation. J. Math. Mech. 16, 1361-1376 (1967)

[33] Mironescu, P., Rădulescu, V.: A bifurcation problem associated to a convex, asymptotically linear function. C. R. Acad. Sci. Paris, Sér. I Math. 316(7), 667-672 (1993)

[34] Mironescu, P., Rădulescu, V.: The study of a bifurcation problem associated to an asymptotically linear function. Nonlinear Anal. 26(4), 857-875 (1996)

[35] Molino, A.: Gelfand type problem for singular quadratic quasilinear equations. NoDEA Nonlinear Diff. Equ. Appl. 23(5), 23-56 (2016)

[36] Nedev, G.: Regularity of the extremal solution of semilinear elliptic equations. C. R. Acad. Sci. Paris, Sér. I Math. 330, 997-1002 (2000)

[37] Orsina, L., Puel, J.P.: Positive solutions for a class of nonlinear elliptic problems involving quasilinear and semilinear terms. Comm. Partial Diff. Equ. 26, 16651689 (2001)

[38] Stampacchia, G.: Équations Élliptiques du Second Ordre à Coefficients Discontinus, Séminaire de Mathématiques Supérieures, No. 16 (Été, 1965), Les Presses de l'Université de Montréal, Montréal, (1966)

[39] Terra, J.: Stable solutions of equations with a quadratic gradient term. Electron. J. Diff. Equ. 2016(196), 1-22 (2016)

Asadollah Aghajani and Fatemeh Mottaghi

School of Mathematics

Iran University of Science and Technology

Narmak

Tehran

Iran

e-mail: aghajani@iust.ac.ir

Fatemeh Mottaghi

e-mail: mottaghi@mathdep.iust.ac.ir

Vicenţiu D. Rădulescu

Faculty of Applied Mathematics

AGH University of Science and Technology

30-059 Krakow

Poland

and 
Department of Mathematics

University of Craiova

200585 Craiova

Romania

e-mail: radulescu@inf.ucv.ro

and

Simion Stoilow Institute of Mathematics of the Romanian Academy 21 Calea Grivitei Street

010702 Bucharest

Romania

Received: August 31, 2019.

Revised: March 25, 2020.

Accepted: October 6, 2020. 